\title{
Optimization Methods for Power Allocation and Interference Coordination Simultaneously with MIMO and Full Duplex for Multi-Robot Networks
}

\author{
Guisheng Wang ${ }^{1 *}$, Yequn Wang ${ }^{1,2}$, Shufu Dong ${ }^{1}$, Guoce Huang ${ }^{1}$, and Qilu Sun ${ }^{1}$ \\ ${ }^{1}$ Information and Navigation College, Air Force Engineering University \\ Xi' an, 710077, China \\ [e-mail:wgsfuyun@163.com] \\ ${ }^{2}$ The 30th Research Institute of China Electronics Technology \\ Chengdu, 610054, China \\ [e-mail: kgdwyq@126.com,kgddsf@126.com,kgdhgc@126.com,kgdwyb@163.com] \\ *Corresponding author: Guisheng Wang
}

Received August 28, 2020; revised November 16, 2020; accepted December 25, 2020; published January 31, 2021

\begin{abstract}
The present work addresses the challenging problem of coordinating power allocation with interference management in multi-robot networks by applying the promising expansion capabilities of multiple-input multiple-output (MIMO) and full duplex systems, which achieves it for maximizing the throughput of networks under the impacts of Doppler frequency shifts and external jamming. The proposed power allocation with interference coordination formulation accounts for three types of the interference, including cross-tier, co-tier, and mixed-tier interference signals with cluster head nodes operating in different full-duplex modes, and their signal-to-noise-ratios are respectively derived under the impacts of Doppler frequency shifts and external jamming. In addition, various optimization algorithms, including two centralized iterative optimization algorithms and three decentralized optimization algorithms, are applied for solving the complex and non-convex combinatorial optimization problem associated with the power allocation and interference coordination. Simulation results demonstrate that the overall network throughput increases gradually to some degree with increasing numbers of MIMO antennas. In addition, increasing the number of clusters to a certain extent increases the overall network throughput, although internal interference becomes a severe problem for further increases in the number of clusters. Accordingly, applications of multi-robot networks require that a balance should be preserved between robot deployment density and communication capacity.
\end{abstract}

Keywords: Multiple-input, Multiple-Output, Full Duplex, Interference Coordination, Power Allocation, Iterative Optimization, Multi-Robot Networks

This research was supported by a research grant from the National Natural Science Foundation of China [61701521], and The Postdoctoral Science Foundation of China [2016M603044]. We express our thanks to Dr. Mohammed Atiquzzaman for the constructive suggestions. 


\section{Introduction}

Explosive growth in the use of collaborative groups of multi-robots has occurred over the past two decades in applications such as reconnaissance and surveillance [1], patrol [2], and search and rescue [3] tasks, which have increasingly depended on the reliable capability of exchanging data between the individual robot entities via wireless multi-robot networks [4]. Moreover, establishing reliable and continuous communication links between each cluster of multi-robots is essential for ensuring rapid deployment and flexible configuration and reconfiguration of robot clusters during mission execution, especially in the disaster areas or battlefield. Multi-robot networks are further essential for ensuring the exchange of information between clusters when clusters regroup, and for supporting data transmission with centralized facilities via downlinks and uplinks [5].

However, among the most critical issues confronting multi-robot networks are the restricted energy capacity of robot communication devices and the means of efficiently managing their power consumption [6]. These issues are denoted as resource sharing problems in energy, time, and network frequency spectrum [7, 8]. Here, energy consumption in multi-robot networks is not only subjected to power amplification and signal processing tasks, but, to a major extent, is also subjected to additional complications owing to the motion of robots. In particular, the limited range of communication devices results in increasing power consumption with increasing distance between robots owing to diminishing signal strength and increasing interference. As a result, a power consumption threshold must be applied to manage power consumption, which can result in the loss of network connectivity with increasing robot distance $[9,10]$. In addition, the frequency spectrum of multi-robot networks must be strictly limited to insure inter-network independence. Accordingly, the number of channels is few, particularly for broadband systems, and these results in co-channel interference in the form of transmission collisions that occur when two concurrent transmissions lie within the time-varying interference range of each link, and result in the failure of both transmissions. Accordingly, energy efficiency and frequency spectrum efficiency in wireless energy-constrained multi-robot networks have generated increasing attention recently.

Power control is an important method employed in interference management, and its adoption can mitigate interference as well as reduce energy consumption. Observations regarding the inducement of collisions and the opportunities for concurrent transmission in wireless networks were employed to develop an efficient interference-aware power control protocol with increased network throughput $[11,12]$. However, existing studies focus on both resource allocation and interference coordination, the hot topics are mainly developed for heterogeneous networks, particularly in dense scenarios of $5 \mathrm{G}$ technologies. The limitations of conventional technology make it difficult to achieve further ascension of network capacity and spectrum or energy efficiency. An analysis of the effects of co-channel interference in frequency-hopping ad hoc inter-networks employing synchronized cooperative broadcast demonstrated that co-channel interference in such networks significantly degraded network throughput [13]. A low-complexity spectrum allocation scheme was proposed for constraining co-channel interference in a cognitive radio network, and the approach simultaneously achieved efficient power control and network throughput [14]. Another study demonstrated that multi-robot networks should maximize their energy efficiency to accommodate the limited onboard energy of robot communication devices, and thereby improve the quality of 
communication between multi robot entities [15]. In addition, an energy-efficient bandwidth allocation scheme was proposed for wireless networks to meet quality of service (QoS) demands during resource allocation [16].

We note that gaps exist between studies focused on power allocation and interference coordination problems in multi-robot networks [17]. These are particularly challenging tasks to address for multi-robot networks because interference is a critical problem influencing the communications between robots, and thereby negatively affecting collaboration between groups of networking robot entities, which becomes more severe when the communication is dominated by line of sight (LoS) channels [18]. Moreover, the mobility of multiple robot entities can induce Doppler shifts in the transmitted signals, which generates severe interference at higher communication frequencies. Also, adjacent clusters of robot entities are subject to interference from each other during uplink and downlink network communications [19]. Therefore, the problem of interference can be mitigated by diversifying the frequency spectrum or by enabling efficient coordination among the communication transmissions in multi-robot networks [20].

The present study addresses the above-discussed issues in multi-robot networks by proposing a group-centric network organization that applies the promising expansion capabilities of multiple-input multiple-output (MIMO) and full duplex systems for maximizing the throughput of the network under the impacts of Doppler frequency shifts and external jamming. The primary contributions of the present study are summarized as follows.

1) A two-tier group-centric hierarchical framework is adopted for maximizing the overall throughput of the multi-robot network. The framework includes a single centralized processing node (CPN) with $N$ MIMO antennas, cluster head nodes (CHNs), and scattered cluster member nodes (CMNs). The issues associated with MIMO are addressed by equipping each of the individual robot entities with a single antenna operating in full-duplex communication modes. Here, we take advantage of the ability of each robot to operate in different full-duplex modes by adopting two mode types, including an out-of-band full-duplex (OBFD) mode, where the uplink and downlink are conducted in orthogonal channels, and an in-band full-duplex (IBFD) mode, where the uplink and downlink channels are in the same frequency band. In other words, the individual robots may engage in transmitting and receiving in distinct frequency or co-frequency modes simultaneously.

2) The proposed power allocation with interference coordination formulation accounts for three types of the interference, including cross-tier, co-tier, and mixed-tier interference signals with CHNs operating in both OBFD and IBFD modes, and their signal-to-noise-ratios (SNRs) are respectively derived under the impacts of Doppler frequency shifts and external jamming.

3) Preliminary investigation using five different optimization algorithms revealed that the iterative call-back optimization algorithm provided the best optimization performance based on its computational speed and the high performance of the obtained network.

The remainder of this paper is organized as follows. Section 2 describes the hierarchical network, communication and control technologies in the related work. Section 3 introduces the full-duplex downlink transmission scheme for CPNs, and derives approximations of the SNR values obtained under cross-tier, co-tier, and mixed-tier interference signals between the $\mathrm{CPN}$ and CHNs operating in OBFD and IBFD modes. These factors are then combined to formulate the proposed power allocation with interference coordination problem. Section 4 presents the centralized and decentralized optimization algorithms proposed for solving the power allocation with interference coordination problem. Then, the results of simulations are analyzed in Section 5 to investigate the impacts of the number of MIMO antennas employed in the $\mathrm{CPN}$ of the network and the number of clusters employed within the network on network 
throughput. Finally, the conclusions of the study are drawn in Section 6.

\section{Related Work}

\subsection{Hierarchical Network}

Multi-robot networks that accommodate multiple states will improve the environmental adaptability of the network by accounting for the dynamically varying characteristics of diverse robots with respect to communication modes, waveforms, network operations, and capabilities. Therefore, a common framework is conducive to the integration of heterogeneous networks, and applying a group-centric approach will increase the scope of network applications. Accordingly, the group-centric framework illustrated in Fig. $\mathbf{1}$ is adopted, which presents an overall structure having the characteristics of heterogeneity, self-organization, and decentralized control. Here, wireless communication is provided by the single CPN with $N$ MIMO antennas, while the individual robot entities are still equipped with single antennas operating in full-duplex modes.

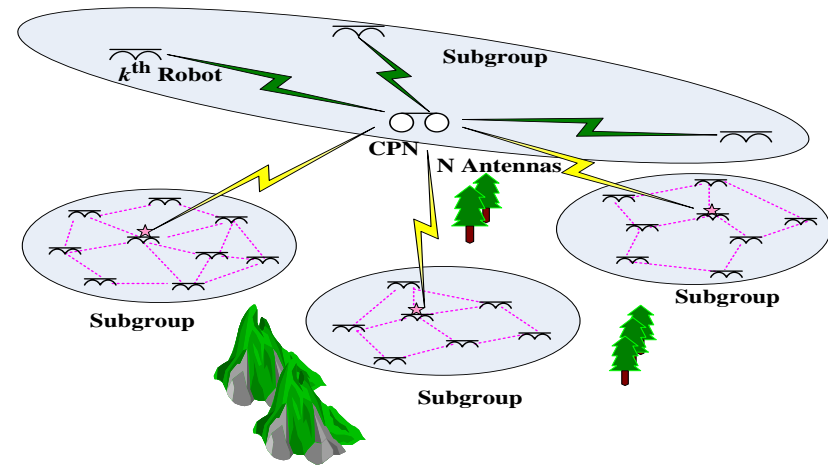

Fig. 1. Overall group-centric framework of the proposed multi-robot network including the centralized processing node (CPN) with $N$ MIMO antennas communicating with subgroups

The two-tier hierarchical group-centric mapping structure illustrated in Fig. $\mathbf{2}$ is adopted to achieve distributed and grouped communications. Network management in the first tier of sub-groups operates via intra-network communication through the CPN and CHNs, and that in the second tier operates via inter-network communication between CHNs and CMNs. Moreover, the nodes in the second tier are generated with different function domains by $S$ clusters in the first tier via either adaptive election or mandatory assignment according to the different requirements and characteristics of the multi-robot network. In addition, the decentralized subgroups adopt self-organizing modes for $K$ robot entities, which is convenient for sharing information randomly. 


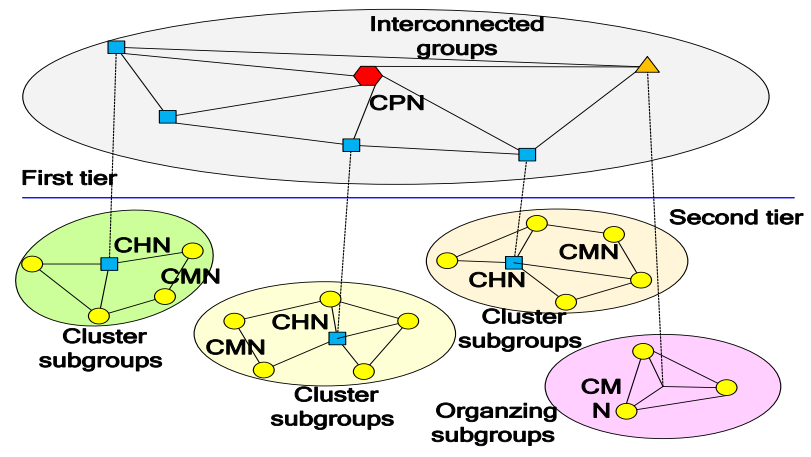

Fig. 2. Two-tier hierarchical mapping structure of the proposed multi-robot network including cluster head nodes (CHNs) and cluster member nodes (CMNs)

\subsection{MIMO \& Full-duplex}

Further advancements in the transmission capacity of wireless communication networks are applicable toward enhancing the performance of multi-robot networks. For example, MIMO technology is a promising method for increasing the transmission capacity of mobile communications systems by exploiting multipath communications between multiple transmission and receiving antennas to reduce latency, enhance energy efficiency, and mitigate channel fade [21, 22]. In fact, Heliot et al. [23] demonstrated that MIMO technology can improve the spectrum efficiency and energy efficiency of wireless networks simultaneously. The developed network based on MIMO was demonstrated to outperform conventional networks whereas the existing methods are not incommensurate with the dynamic deployment and flexible configuration in the multi-robot networks.

We also note that the development of $5 \mathrm{G}$ technology has demonstrated the advantages of full-duplex communication systems in terms of high communication rate and spectrum utilization [24]. Here, full-duplex wireless nodes have a demonstrated capability for accommodating simultaneous transmission and reception in the same frequency band, therefore they can effectively double the data transmission rate [25]. In addition, Do et al. [26] developed a power-time allocation scheme for a full-duplex wireless powered communication network based on residual self-interference subject to energy causality constraints.

\subsection{Mobility \& Coordination}

The present work focuses on aerial robots, such as UAVs (also known as drones), owing to their high mobility and flexible deployment in three-dimensional (3D) space. A region of 3D space can be explored randomly by multiple aerial robots with dynamically varying locations and velocities, based on a random mobility model like the random waypoint (RWP) model [27]. However, most mobility models intended specifically for multiple aerial robot entities are mission-based control patterns, and the connectivity-oriented control models employed assume that network connectivity is self-organizing with only local information [28]. In this paper, multiple robot entities can adjust their locations or trajectories to maintain favorable LoS channels based on the locations and/or movements of the CPN or CHNs. Moreover, resource management in the proposed multi-robot network is conducted based on two different control methods, including centralized management and distributed optimization. In centralized management, the CPN acts as a centralized processing center to control the CHNs, and the CHNs manage the power and frequency resources in the responding subgroups, which achieves global, hierarchical, and direct resource management. Distributed optimization is employed when unforeseeable problems arise in scattered CMNs, CHNs, or the CPN to 
recover good communication performance by implementing a local self-optimization function from the bottom up, which overcomes bottleneck constraints in the overall network.

\subsection{Communication \& Interference}

We note that existing quantization errors and coupling effects will lead to instability in multi-robot networks. Accordingly, a range of complex properties deserves to achieve efficient information processing and functional interactions between the network nodes. The relaying retransmission forward and the active learning route conduct as propellers. Moreover, error correction and increased signal amplification can be utilized to further improve the reliability of communications in practical applications. In addition, the communication process of multi-robot networks requires high-rate and wideband data transmission, which introduces specific interference coordination problems, particularly for intra-tier and inter-tier communications between sub-groups using frequency-division duplex (FDD) modes.

In this paper, the CPN utilizes a zero-forcing (ZF) precoding method to eliminate self-interference in transmission caused by multiple antenna channels. While these channels are mainly dominated by LoS components, limited multipath fade may also be present due to ground reflections, where the effect is mainly based on path loss. At the same time, the high mobility of the robots indicates that the channels may be affected by Doppler frequency shifts, which will affect spectrum allocation directly. In consequence, we employ the Clarke flat fade channel model [29] to approximate the influence of Doppler frequency shifts and consider external jamming as well. The complexity of the communication system is reduced by assuming that all transmissions in the multi-robot network are completely synchronized, and the CPN can obtain accurate channel state information (CSI) in the time-division duplex (TDD) mode by means of channel reciprocity. Furthermore, each sub-group implements the FDD mode to improve the overall throughput of the network.

\section{System Model \& Problem Formulation}

\subsection{Full-Duplex Modes}

According to the literatures on full-duplex, each robot can operate in different full-duplex modes, which will be categorized into two types broadly: out-of-band full-duplex OBFD (the uplink and downlink are conducted in orthogonal channels) and in-band full-duplex IBFD (the uplink and downlink channels are in the same frequency band). In other words, they may refer to transmitting and receiving in distinct frequency or co-frequency modes simultaneously.

Considering that the CPN can communicate wirelessly with the CHNs and CMNs simultaneously, different spectrum allocation methods will lead to diverse interference coordination problems in the transmission process. We address this issue by dividing the available frequency band into two components $F_{1}$ and $F_{2}$, and assume that the uplink channel is $F_{1}$ and the downlink channel is $F_{2}$ [30]. As such, the selection of $F_{1}$ or $F_{2}$ for channel modes will directly determine the full duplex modes corresponding to the CHNs and CMNs, which generates different types of co-tier and cross-tier interference, as illustrated in Fig. 3. If a CHN selects $\mathrm{F}_{1}$ as the uplink channel, then the CHN works in the OBFD mode, and if a CHN selects $\mathrm{F}_{2}$ as the downlink channel, then the CHN works in the IBFD mode. In this case, we refer to the set of nodes working in the OBFD mode as $S_{\mathrm{O}}$, and the other nodes working in the IBFD mode as $S_{\mathrm{I}}$, where $\left|S_{\mathrm{O}} \cup S_{\mathrm{I}}\right|=S$, and $S_{\mathrm{O}} \cap S_{\mathrm{I}}=\varnothing$. 

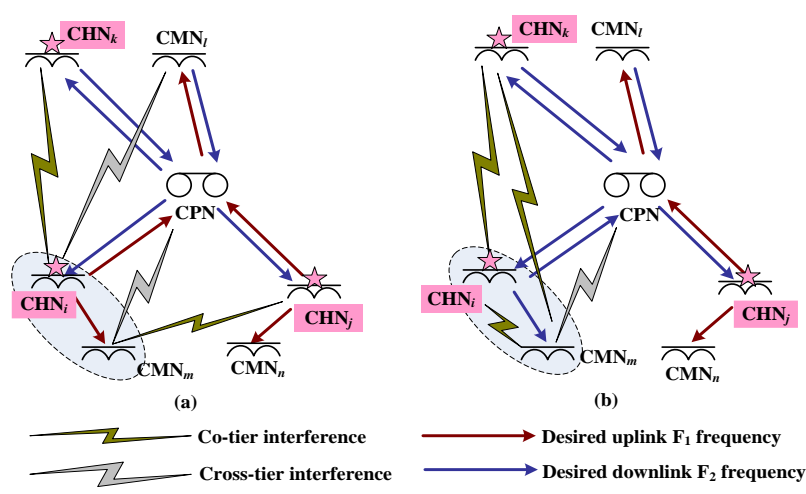

Fig. 3. Interference types in the hierarchical mapping structure of the multi-robot network: (a) interference for downlinks in the out-of-band full-duplex (OBFD) mode (b) interference for downlinks in the in-band full-duplex (IBFD) mode

The present work determines network throughput based on the capacities of the downlink channels because when the TDD mode is employed by the CPN, and the capacity requirement of the downlink channels is much greater than that of the uplink channels. According to the preceding discussion, network interference can be divided into three components, including cross-tier, co-tier, and mixed-tier interference signals, which depend upon the CHNs in sets $S_{\mathrm{O}}$ and $S_{\mathrm{I}}$.

\subsection{Interference Coordination}

\subsubsection{Cross-Tier Interference}

This subsection considers the cross-tier interference arising at $\mathrm{CMN}_{l}$ from channel $\mathrm{F}_{1}$ of $\mathrm{CHN}_{i}$ in Fig. 3(a) when some CHNs operate in the OBFD mode, which exist in set $S_{\mathrm{O}}$, then the OBFD modes are also selected in the corresponding subgroups. Here, the signal received by $\mathrm{CMN}_{l}$ can be expressed as

$$
y_{C M N_{l}}=\boldsymbol{h}_{P 2 M_{l}} \boldsymbol{W} x_{P 2 M_{l}}(t)+\sum_{i \in S_{O}}\left[\boldsymbol{h}_{H_{i} 2 M_{l}} x_{H_{i} 2 M_{l}}(t)\right]+j(t)+n(t)
$$

where $\boldsymbol{h}_{P 2 M_{l}} \in \mathbf{C}^{1 \times N}$ and $\boldsymbol{h}_{H_{i} 2 M_{l}} \in \mathbf{C}$ represent the fading channel matrix with path loss from the $\mathrm{CPN}$ to $\mathrm{CMN}_{l}$, respectively, and the Doppler frequency shifts from CHNs to $\mathrm{CMN}_{l}$, where all of them are accordance with the complex domain $\mathbf{C}, \boldsymbol{W} \in \mathbf{C}^{N \times K}$ is a precoding matrix for the CPN communicating with CMNs, $x_{P 2 M_{l}}(t) \sim C N\left(0, P_{C P} / K\right)$ and $x_{H_{i} 2 M_{l}}(t) \sim C N\left(0, P_{\mathrm{CH}}\right)$ are signals in compliance with the complex normal distributions of the transmission power parameter $P_{\mathrm{CP}}$ in the CPN and the transmission power parameter $P_{\mathrm{CH}}$ in CHNs, respectively, and $j(t)$ and $n(t)$ respectively represent an external jamming signal with a variance of $\sigma_{J}^{2}$ and natural Gaussian noise with a variance of $\sigma_{N}{ }^{2}$. Therefore, the SNR of $\mathrm{CMN}_{l}$ is simply given as

$$
r_{C M N_{l}}=\frac{P_{C P}\left\|\boldsymbol{h}_{P 2 M_{l}} W\right\|^{2}}{K\left[\sum_{i \in S_{O}} P_{C H}\left\|\boldsymbol{h}_{H_{i} 2 M_{l}}\right\|^{2}+\sigma_{J}^{2}+\sigma_{N}^{2}\right]}
$$


The SNR values of the other CMNs under these circumstances can be similarly derived.

\subsubsection{Co-Tier Interference}

Considering the diverse full duplex modes operated in CHNs, we firstly consider the co-tier interference arising at $\mathrm{CHN}_{i}$ from channel $\mathrm{F}_{2}$ of $\mathrm{CHN}_{k}$ in Fig. 3(a) when some CHNs operate in the OBFD mode, which exist in set $S_{\mathrm{O}}$, then the OBFD modes are also selected in the corresponding subgroups, and $\mathrm{CHN}_{k}$ is in $S_{\mathrm{I}}$. Hence, the signal received by $\mathrm{CHN}_{i}$ is

$$
y_{C H N_{i}}^{O}=\boldsymbol{h}_{P 2 H_{i}} \boldsymbol{G} x_{P 2 H_{i}}(t)+\sum_{k \in S_{I}} \boldsymbol{h}_{C_{i} 2 C H_{k}} x_{C_{i} 2 C H_{k}}(t)+j(t)+n(t)
$$

where $\boldsymbol{h}_{P 2 H_{i}} \in \mathbf{C}^{1 \times N}$ and $\boldsymbol{h}_{\mathrm{CH}_{i} 2 \mathrm{CH}_{k}} \in \mathbf{C}$ respectively represent the fading channel matrixes between the CPN and $\mathrm{CHN}_{i}$, and $\mathrm{CHN}_{i}$ and $\mathrm{CHN}_{k}, \boldsymbol{G} \in \mathbf{C}^{N \times S}$ is a precoding matrix for the $\mathrm{CPN}$ communicating with $\mathrm{CHNs}, x_{P_{22 H_{i}}}(t) \sim C N\left(0, P_{C P} / S\right)$ and $x_{\mathrm{CH}_{i} 2 \mathrm{CH}_{k}}(t) \sim C N\left(0, P_{\mathrm{CH}}\right)$ represent signals obeying the complex normal distributions of parameters $P_{\mathrm{CP}}$ and $P_{\mathrm{CH}}$, respectively. Hence, the SNR of $\mathrm{CHN}_{i}$ in OBFD mode is

$$
r_{C H N_{i}}^{O}=\frac{P_{C P}\left\|\boldsymbol{h}_{P 2 H_{i}} \boldsymbol{G}\right\|^{2}}{S\left[P_{C H} \sum_{k \in S_{I}}\left\|\boldsymbol{h}_{C H_{i} 2 C H_{k}}\right\|^{2}+\sigma_{J}^{2}+\sigma_{N}^{2}\right]}
$$

On the contrary, when some CHNs operate in the IBFD mode, which exist in set $S_{\mathrm{I}}$, then the IBFD modes are also selected in the corresponding subgroups, and $\mathrm{CHN}_{\mathrm{k}}$ is in $S_{\mathrm{I}}$. As such, we consider the co-tier interference arising at $\mathrm{CHN}_{i}$ from channel $\mathrm{F}_{2}$ of $\mathrm{CHN}_{k}$ in $\mathbf{F i g} . \mathbf{3}(\mathbf{b})$, and the signal received by $\mathrm{CHN}_{i}$ is given as follows.

$$
\begin{aligned}
y_{C H N_{i}}^{I}= & \boldsymbol{h}_{P 2 H_{i}} \boldsymbol{G} x_{P 2 H_{i}}(t)+\sum_{k \in S_{I} \backslash i} \boldsymbol{h}_{C_{i} 2 C H_{k}} x_{C^{2} H_{i} 2 H_{k}}(t) \\
& +\boldsymbol{h}_{\mathrm{CH}_{i} 2 \mathrm{CM}_{\text {in }}} x_{\mathrm{CH}_{i} 2 C M_{\text {im }}}(t)+j(t)+n(t)
\end{aligned}
$$

Here, $\boldsymbol{h}_{\mathrm{CH}_{i} 2 \mathrm{CM}_{\text {in }}} \in \mathbf{C}$ represents the fading channel matrix between $\mathrm{CHN}_{i}$ and $\mathrm{CMNs}$ in the same subgroup, and $x_{\mathrm{CH}_{i} 2 \mathrm{CM}_{\text {in }}}(t) \sim C N\left(0, P_{\mathrm{CM}}\right)$ is a signal subject to the complex normal distribution of parameter $P_{\mathrm{CM}}$ in CMNs. Based on this model, the $\mathrm{SNR}$ of $\mathrm{CHN}_{i}$ in IBFD mode is given by

$$
r_{C H N_{i}}^{I}=\frac{P_{C P}\left\|\boldsymbol{h}_{P 2 H_{i}} \boldsymbol{G}\right\|^{2}}{S\left[P_{C H} \sum_{k \in S_{I} \backslash i}\left\|\boldsymbol{h}_{C H_{i} 2 C H_{k}}\right\|^{2}+P_{C M}\left\|\boldsymbol{h}_{C H_{i} 2 C M_{\text {im }}}\right\|^{2}+\sigma_{J}^{2}+\sigma_{N}^{2}\right]}
$$




\subsubsection{Mixed Interference}

Considering the diverse full duplex modes operated in CHNs, we firstly consider the mixed interference at $\mathrm{CMN}_{m}$ due to the cross-tier interference arising from channel $\mathrm{F}_{2}$ of the CPN and the co-tier interference arising from channel $\mathrm{F}_{1}$ of $\mathrm{CHN}_{j}$ in $S_{\mathrm{O}}$, as shown in Fig. 3(a), when some CHNs exist in set $S_{\mathrm{O}}$. Therefore, the signal received by $\mathrm{CMN}_{m}$ is given as follows.

$$
\begin{aligned}
y_{C H N_{m}}^{O}= & \boldsymbol{h}_{C H_{i} 2 C M_{i m}} x_{C H_{i} 2 C M_{\text {im }}}(t)+\sum_{j \in S_{O} \backslash i} \boldsymbol{h}_{C H_{j} 2 C M_{\text {im }}} x_{C H_{j} 2 C M_{\text {in }}}(t) \\
& +\boldsymbol{h}_{P 2 H_{i}} \boldsymbol{W} x_{M 2 H_{i}}(t)+j(t)+n(t)
\end{aligned}
$$

Here, $\boldsymbol{h}_{\mathrm{CH}_{i} 2 \mathrm{CM}_{i n}} \in \mathbf{C}$ and $\boldsymbol{h}_{\mathrm{CH}_{j} 2 \mathrm{MM}_{i n}} \in \mathbf{C}$ respectively represent the fading channel matrixes for $\mathrm{CMN}_{m}$ connecting with $\mathrm{CHN}_{i}$ in the same subgroup and connecting with $\mathrm{CHN}_{j}$ in the other subgroup, and $x_{\mathrm{CH}_{i} 2 C M_{\text {in }}}(t) \sim C N\left(0, P_{\mathrm{CH}}\right)$ and $x_{\mathrm{CH}_{j} 2 \mathrm{CM}_{i n}}(t) \sim C N\left(0, P_{\mathrm{CH}}\right)$ are signals subject to the complex normal distributions of parameter $P_{\mathrm{CH}}$. We note here that the cross-tier interference in CMNs generated by the CPN can be eliminated by applying the forced-zero precoding method. Therefore, the mixed interference SNR of $\mathrm{CMN}_{m}$ can be defined by

$$
r_{C H N_{m}}^{O}=\frac{P_{C H}\left\|\boldsymbol{h}_{C_{i} 2 C M_{i n}}\right\|^{2}}{\left[P_{C H} \sum_{j \in S_{O} \backslash i}\left\|\boldsymbol{h}_{C H_{j} 2 C M_{i n}}\right\|^{2}+\sigma_{J}^{2}+\sigma_{N}^{2}\right]}
$$

On the contrary, when some CHNs exist in set $S_{\mathrm{I}}$, we consider the mixed interference at $\mathrm{CMN}_{m}$ due to the cross-tier interference arising from channel $\mathrm{F}_{2}$ of the CPN and the co-tier interference arising from channel $\mathrm{F}_{2}$ of $\mathrm{CHN}_{k}$ in $S_{\mathrm{I}}$, as shown in Fig. 3(b), Therefore, the signal received by $\mathrm{CMN}_{m}$ is given as follows.

$$
\begin{aligned}
y_{C H N_{m}}^{I}= & \boldsymbol{h}_{C H_{i} 2 C M_{\text {in }}} x_{C H_{i} 2 C M_{\text {in }}}(t)+\sum_{k \in S_{I} \backslash i} \boldsymbol{h}_{C H_{k} 2 C M_{\text {in }}} x_{C H_{k} 2 C M_{\text {in }}}(t) \\
& +\boldsymbol{h}_{P 2 H_{i}} \boldsymbol{G} x_{M 2 H_{i}}(t)+j(t)+n(t)
\end{aligned}
$$

Here, $\boldsymbol{h}_{\mathrm{CH}_{k} 2 \mathrm{CM}_{\text {in }}} \in \mathbf{C}$ represents the fading channel matrix between $\mathrm{CHN}_{k}$ in the other subgroup and $\mathrm{CMN}_{m}$, and $x_{\mathrm{CH}_{k} 2 C M_{\text {in }}}(t) \sim C N\left(0, P_{\mathrm{CH}}\right)$ represents a signal obeying the complex normal distribution of parameter $P_{\mathrm{CH}}$. Similarly, the cross-tier interference in CMNs generated by the CPN can be eliminated by applying the zero-forcing precoding method. Therefore, the $\mathrm{SNR}$ of $\mathrm{CMN}_{m}$ is defined as

$$
r_{C H N_{m}}^{I}=\frac{P_{C H}\left\|\boldsymbol{h}_{C H_{i} 2 C M_{\text {in }}}\right\|^{2}}{\left[P_{C H} \sum_{k \in S_{I} \backslash i}\left\|\boldsymbol{h}_{C H_{k} 2 C M_{i n}}\right\|^{2}+\sigma_{J}^{2}+\sigma_{N}^{2}\right]}
$$




\subsection{Simultaneous Power Allocation and Interference Coordination}

The complicated co-tier and cross-tier interference problems associated with the different mode selections of CHNs and CMNs make it necessary to assign modes to the network nodes in advance. Therefore, we model the interference coordination problem as a specific optimization problem using 0-1 integer programming to maximize the overall throughput of the network. According to Shannon's theorem, the overall throughput of a multi-robot network can be obtained as follows.

$$
\begin{aligned}
C & =C_{C M N}+C_{C H N}^{O}+C_{C H N}^{I} \\
& =\sum_{k \in K} B_{M_{k}} \log _{2}\left(1+r_{C M N_{k}}\right)+\sum_{i \in S_{O}} B_{H_{i}} \log _{2}\left(1+r_{C H N_{i}}^{O}\right)+\sum_{j \in S_{I}} B_{H_{j}} \log _{2}\left(1+r_{C H N_{j}}^{I}\right)
\end{aligned}
$$

Here, the throughputs $C_{C M N}, C_{C H N}^{O}$, and $C_{C H N}^{I}$ represent those of CMNs for the CPN operating in the TDD mode, those of CHNs operating in OBFD modes, and those of CHNs operating in IBFD modes, respectively, while $B_{M k}, B_{H i}$, and $B_{H j}$ respectively denote the channel bandwidths for the $k^{\text {th }} \mathrm{CMN}$, and the $i^{\text {th }}$ and $j^{\text {th }}$ CHNs. Then, we set a vector $\boldsymbol{x}=\left[x_{1}, x_{2}, \cdots, x_{S}\right]^{T}$ to denote the full duplex modes of CHNs, where $x_{i}$ is a binary variable with the following connotations.

$$
x_{i}= \begin{cases}0, & \mathrm{CHN}_{i} \text { is operated in OBFD mode } \\ 1, & \mathrm{CHN}_{i} \text { is operated in IBFD mode }\end{cases}
$$

We also set a variable $w_{i}$ for adjusting the power allocation in the $i^{\text {th }} \mathrm{CHN}$ :

$$
\left\{\begin{array}{l}
P_{C H}^{i}=w_{i} P_{C H}, P_{C H}^{j}=w_{j} P_{C H} \\
0 \leq w_{i}, w_{j} \leq 1, w_{i}, w_{j} \in R
\end{array}\right.
$$

This yields the power allocation weight vector $\boldsymbol{w}=\left[w_{1}, w_{2}, \cdots, w_{i}, \cdots, w_{S}\right]$ for the $S$ CHNs, which is defined independently of $\boldsymbol{x}$. Therefore, the overall optimization of network throughput problem can be derived as follows.

$$
\begin{aligned}
C & =\sum_{k=1}^{K} B_{M_{k}} \log _{2}\left(1+r_{C M N_{k}}\right)+\sum_{i=1}^{S}\left(1-x_{i}\right) B_{H_{i}} \log _{2}\left(1+r_{C H N_{i}}^{O}\right)+\sum_{i=1}^{S} x_{i} B_{H_{i}} \log _{2}\left(1+r_{C H N_{i}}^{I}\right) \\
= & \sum_{k=1}^{K} B_{M_{k}} \log _{2}\left(1+\alpha_{k} /\left[\sum_{i=1}^{S}\left(1-x_{i}\right) w_{i} \beta_{k i}+\sigma^{2}\right]\right)+\sum_{i=1}^{S}\left(1-x_{i}\right) B_{H_{i}} \log _{2}\left(1+\gamma_{i} /\left[\sum_{j=1}^{S} x_{j} w_{i} \eta_{i j}+\sigma^{2}\right]\right) \\
& +\sum_{i=1}^{S}\left(1-x_{i}\right) B_{H_{i}} \log _{2}\left(1+\varphi_{i} /\left[\sum_{j=1}^{S}\left(1-x_{j}\right) w_{j} \lambda_{i j}+\sigma^{2}\right]\right) \\
& +\sum_{i=1}^{S} x_{i} B_{H_{i}} \log _{2}\left(1+\gamma_{i} /\left[\sum_{j=1}^{S} x_{j} w_{i} \eta_{i j}+v_{i} / w_{i}+\sigma^{2}\right]\right)+\sum_{i=1}^{S} x_{i} B_{C} \log _{2}\left(1+\varphi_{i} /\left[\sum_{j=1}^{S} x_{j} w_{j} \lambda_{i j}+\sigma^{2}\right]\right)
\end{aligned}
$$


Here, the following definitions are applied.

$$
\left\{\begin{array}{l}
\alpha_{k}=\frac{P_{C P}\left\|h_{P 2 M_{k}} W\right\|^{2}}{K}, \beta_{k i}=P_{C H}\left\|h_{H_{i} 2 M_{k}}\right\|^{2}, \sigma^{2}=\sigma_{J}^{2}+\sigma_{N}^{2} \\
\gamma_{i}=\frac{P_{C P}\left\|h_{P 2 H_{i}} G\right\|^{2}}{S}, \eta_{i j}= \begin{cases}P_{C H}\left\|h_{C H_{i} 2 C H_{j}}\right\|^{2}, & j \neq i \\
0 & , j=i\end{cases} \\
\varphi_{i}=\left\|h_{C H_{i} 2 C M_{i m}}\right\|^{2}, \lambda_{i j}=\left\{\begin{array}{ll}
P_{C H}\left\|h_{C H_{j} 2 C M_{i k}}\right\|^{2} & , j \neq i \\
0 & , j=i
\end{array}, v_{i}=\frac{P_{C M}}{P_{C H}} \varphi_{i}\right.
\end{array}\right.
$$

Obviously, the power allocation with interference coordination problem in (14) is a complex combinatorial optimization problem, and includes a feasible domain with non-convex characteristics. Accordingly, we note that the lack of dependency between $\boldsymbol{x}$ and $\boldsymbol{w}$ simplifies the solution process greatly because the throughput $C(\boldsymbol{x})$ can be optimized separately from $C(w)$ even though the throughput is a function of both $\boldsymbol{x}$ and $\boldsymbol{w}$.

The computational complexity of the proposed simultaneous power allocation and interference coordination scheme includes the following parts:

1) In the first step of $C(\boldsymbol{x}, \boldsymbol{w})$, the complexity of the first accumulation component acquisition is $O(K \cdot S)$ and the remainings are $O\left(S^{2}\right)$, respectively, which are quite fixed.

2) In the second step, since the complexity of 0-1 variable with a tree search in the feasible domain is $O\left(\log _{2}(S)\right)$, and the continunous variable is determined by the convergence speed of the function $C(\boldsymbol{w})$ in the feasible domain whereas it will be obtained the optimum to some degree $\Delta$ and the iterations $T$, which may be regarded as $O(\Delta \cdot T)$.

Hence, the total complexity of the proposed combinatorial optimization problem is no less than in the order of $O\left(K \cdot S \log _{2}(S) \bullet(\Delta \cdot T)\right)$. We note that the traditional optimization method requires an inordinately long time to solve the problem, while the centralized iterative optimization [31] and decentralized optimization algorithms, such as particle swarm optimization (PSO) and steepest descent [32,33], can be considered to be more suitable for solving this power allocation problem compared with conventional optimization methods, which are deserved to be settled further.

\section{Diverse Optimization Algorithms}

\subsection{Centralized Iterative Optimization}

\subsubsection{Iterative Call-back Optimization Algorithm}

Iterative optimization methods [31] provide an optimal solution to non-convex optimization problems, and they can be applied the fast gradient updating for the throughput $C$. Therefore, the problem given in (14) can be simplified by decomposing it into the following two subproblems, and achieve the goal for accelerating the process of optimization in each iteration when the variables $\boldsymbol{x}^{(0)}=\left[x_{1}, x_{2}, \cdots, x_{i}, \cdots, x_{S}\right]$ are temporality fixed. 


$$
\begin{aligned}
& \left\{\begin{array}{l}
\boldsymbol{w}^{*}=\left[w_{1}{ }^{*}, w_{2}{ }^{*}, \cdots, w_{i}^{*}, \cdots w_{S}{ }^{*}\right]=\arg \max _{w} C\left(\boldsymbol{x}^{(0)}, \boldsymbol{w}\right) \\
\text { s.t. }\left\{\begin{array}{l}
0 \leq w_{i}, w_{j} \leq 1 \\
i, j=1,2, \cdots, S
\end{array}\right.
\end{array}\right. \\
& \left\{\begin{array}{l}
\boldsymbol{x}^{*}=\left[x_{1}{ }^{*}, x_{2}{ }^{*}, \cdots, x_{i}{ }^{*}, \cdots, x_{S}{ }^{*}\right]=\arg \max _{\boldsymbol{x}} C\left(\boldsymbol{x}, \boldsymbol{w}^{*}\right) \\
\text { s.t. }\left\{\begin{array}{l}
x_{i} \in\{0,1\} \\
i, j=1,2, \cdots, S
\end{array}\right.
\end{array}\right.
\end{aligned}
$$

Here, $\boldsymbol{w}^{*}$ is first obtained based on an optimization of (14) with respect to $\boldsymbol{w}$ while holding the initial assignments for the full duplex modes of the CHNs $\boldsymbol{x}^{(0)}$ fixed. Then, $\boldsymbol{x}^{*}$ is obtained based on an optimization of (14) with respect to $\boldsymbol{x}$ while holding $\boldsymbol{w}^{*}$ fixed. This process involving (16) and (17) is repeated over multiple iterations until the results satisfy the condition $\boldsymbol{C}^{(t+1)^{*}}-\boldsymbol{C}^{(t)^{*}} \leq \varepsilon$, where $\varepsilon$ represents an arbitrary parameter of precision. During the iterative process, the weight parameters are adjusted using the gradient descent algorithm as follows:

$$
\boldsymbol{w}^{(t+1)^{*}}=\boldsymbol{w}^{(t)^{*}}-\alpha_{t} \nabla C\left(\boldsymbol{x}, \boldsymbol{w}^{(t)^{*}}\right)
$$

where the attenuation rate $\alpha_{t}>0$ is set to ensure that $w$ varies in the range [0,1], and $\nabla C\left(\boldsymbol{x}, \boldsymbol{w}^{(t) *}\right)$ is the gradient of (14). The ICO algorithm is defined in Algorithm 1.

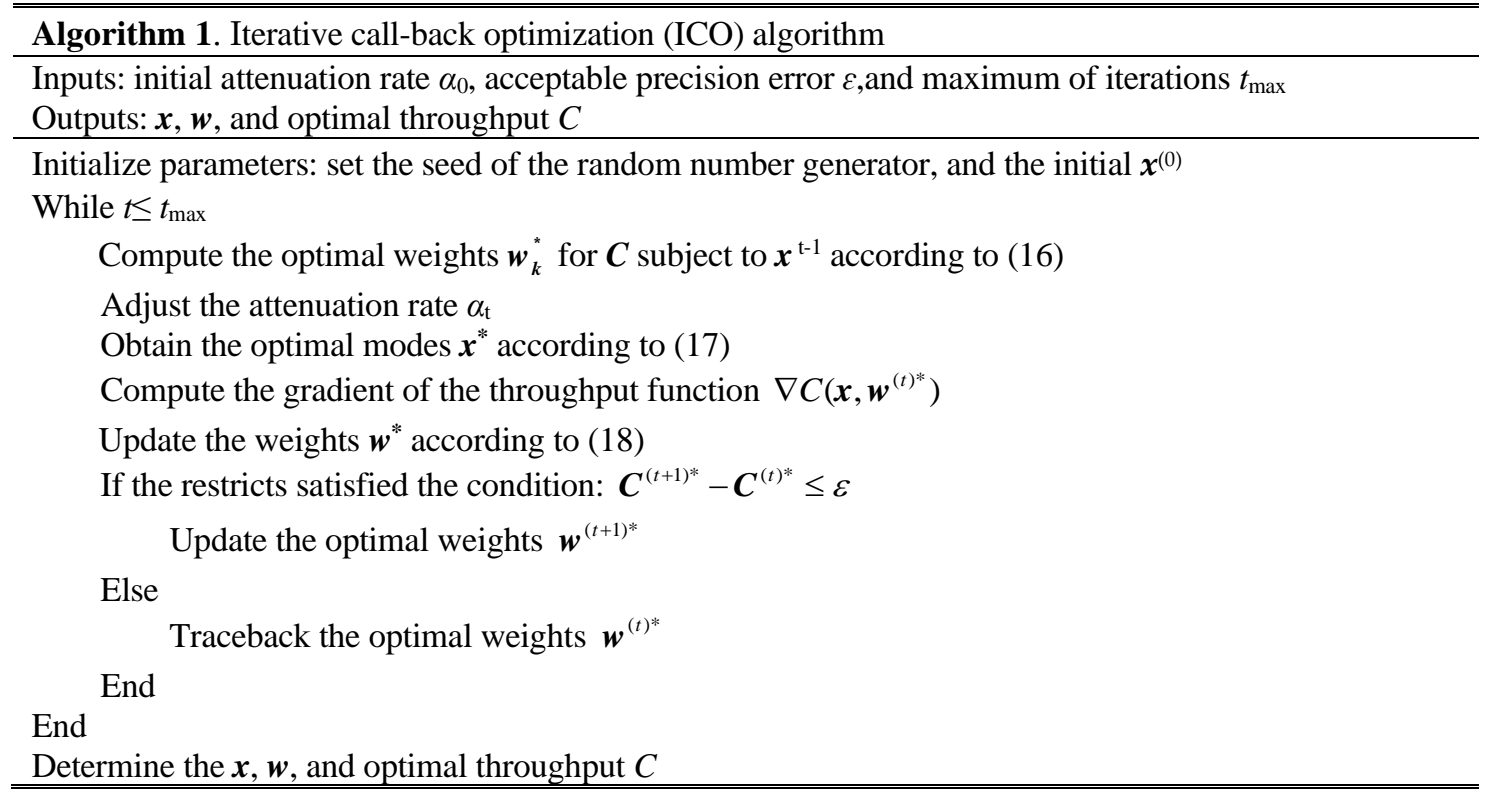

\subsubsection{Iterative Search Algorithm with Steepest Descent}

The iterative search with steepest descent (IS-SD) algorithm similarly decomposes (14) into subproblems involving $\boldsymbol{x}$ and $\boldsymbol{w}$, and searches for optimal solutions along the gradients of $C$ with 
respect to $\boldsymbol{x}$ and $\boldsymbol{w}$, i.e., $\boldsymbol{d}_{x}=-\operatorname{grad}(C, \boldsymbol{x}) ; \boldsymbol{d}_{w}=-\operatorname{grad}(C, \boldsymbol{w})$, until the total gradient $\|\boldsymbol{d}\|_{2}=\left\|\boldsymbol{d}_{x}\right\|_{2}+$ $\left\|\boldsymbol{d}_{w}\right\|_{2}$ is less than an acceptable error value $\varepsilon>0$. The IS-SD algorithm is defined in Algorithm 2.

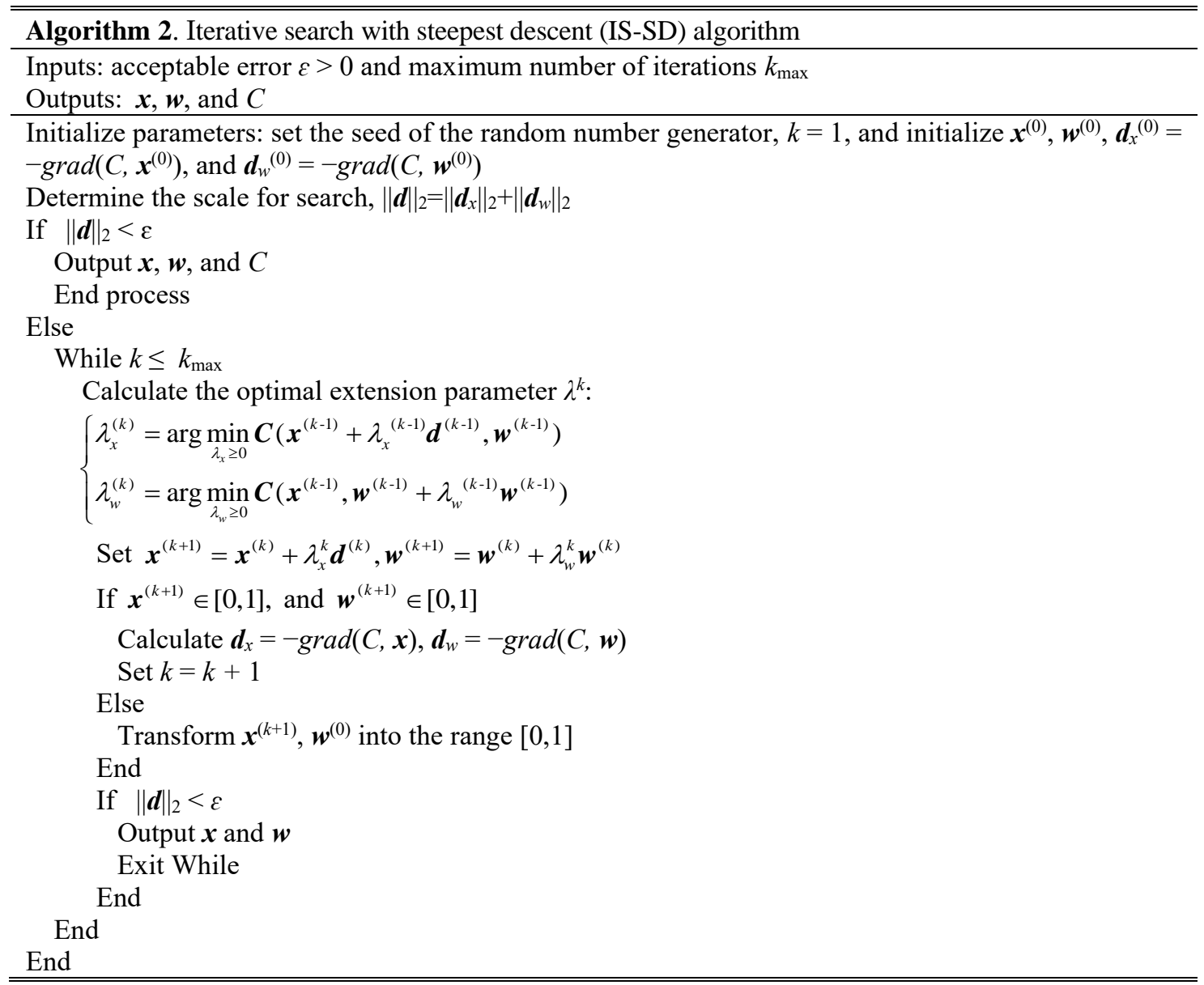

\subsection{Decentralized Swarm Optimization}

\subsubsection{Independent Subspace of Particle Swarm Optimization Algorithm}

The particle swarm optimization (PSO) algorithm $[32,33]$ is newly developed evolutionary technique. Due to the characteristics of efficient search, easy implementation, and quick convergence, nowadays, PSO has gained much attention to diverse issues in different fields, especially for solving multivariable and nonlinear problems. In this section, we bind the concepts of independent subspace in the PSO process to optimize the variables in the network throughput.

The vectors $\boldsymbol{x}$ and $\boldsymbol{w}$ serve as two independent $S$-dimensional search subspaces for the agents or particles employed as a swarm in the PSO. Here, the particles represent candidate solutions that are varied by iteratively moving the particles within the individual search subspaces to obtain $\boldsymbol{x}^{(t)}$ and $\boldsymbol{w}^{(t)}$ values for each particle at the $t^{\text {th }}$ iteration. Then, the throughput $C\left(\boldsymbol{x}^{(t)}, \boldsymbol{w}^{(t)}\right)$ values obtained at the $t^{\text {th }}$ iteration from (14) are compared for all particles individually to determine the best fitness values obtained thus far for each particle. These values are defined as the individual_proportion (or $\boldsymbol{x}_{i}^{(t)}$ ) for $\boldsymbol{x}^{(t)}$ and the individual_weight (or $\left.\boldsymbol{w}_{i}^{(t)}\right)$ for $\boldsymbol{w}^{(t)}$. In addition, the highest fitness values obtained for the individual swarms as a 
whole are defined as the global_proportion $\left(\right.$ or $\boldsymbol{x}_{g}{ }^{(t)}$ ) for $\boldsymbol{x}^{(t)}$ and the global_weight (or $\boldsymbol{w}_{g}{ }^{(t)}$ ) for $\boldsymbol{w}^{(t)}$ until the iterative gradient is less than an acceptable error value $\varepsilon>0$.

The velocity, position, and direction of motion for each agent particle in the search over $\boldsymbol{x}(t)$ are respectively updated as follows.

$$
\begin{aligned}
& \boldsymbol{v}^{(t+1)}=\bmod \left(\boldsymbol{v}^{(t)}+c_{1} \xi_{1}+c_{2} \xi_{2}, 2\right) \\
& \boldsymbol{x}^{(t+1)}=\bmod \left(\boldsymbol{x}^{(t)}+\boldsymbol{v}^{(t+1)}, 2\right)
\end{aligned}
$$

Here, the terms $\boldsymbol{v}^{(0)}, \boldsymbol{x}^{(0)}$, and $\boldsymbol{d}^{(0)}$ are initialized randomly, $\bmod (\cdot)$ represents the general function that returns the decimal portions when one number is divided by another, and the parameters $c_{1}, c_{2}, \xi_{1}$, and $\xi_{2}$ are random variables with normal distributions in the range $[0,1]$. In addition, the direction of motion for each particle in the search over $\boldsymbol{w}^{(t)}$ are updated:

$$
\boldsymbol{d}^{(t+1)}=\omega^{(t)} \boldsymbol{d}^{(t)}+c_{1} \xi_{1}\left(\boldsymbol{w}_{i}-\boldsymbol{w}^{(t)}\right)+c_{2} \xi_{2}\left(\boldsymbol{w}_{g}-\boldsymbol{w}^{(t)}\right) .
$$

Here, the dynamic inertial weight $\omega^{(t)}=\omega_{s}-\left(\omega_{s}-\omega_{e}\right)\left(K_{\max }-k\right) / K_{\max }$, where $\omega_{s}$ is the initial weight, $\omega_{e}$ is the convergent weight of the iteration, and $k$ and $K_{\max }$ are the current iteration number and the maximum number of iterations, respectively. Standard values here are generally $\omega_{s}=0.9$ and $\omega_{e}=0.4$. Considering the motion dynamics and time-sensitivity of the swarms, we also introduce a probe-response mechanism to ensure that the entire population of swarm particles respond to dynamic changes in their external environment by updating the population after detecting changes in the environment. This enables the swarm to appropriately track the dynamic extrema, while avoiding the possibility of the swarm particles falling into local optima. The adaptive environmental updating process is given as follows.

$$
\boldsymbol{w}_{i}^{(t+1)}=\left\{\begin{array}{l}
\frac{1}{t} \boldsymbol{w}_{i}^{(t)}, C\left(x^{(t+1)}, \boldsymbol{w}^{(t+1)}\right)>C\left(x^{(t)}, \boldsymbol{w}^{(t)}\right) \\
\boldsymbol{w}_{i}^{(t+1)}, C\left(x^{(t+1)}, \boldsymbol{w}^{(t+1)}\right) \leq C\left(x^{(t)}, \boldsymbol{w}^{(t)}\right)
\end{array}\right.
$$

The IS-PSO algorithm is defined in Algorithm 3.

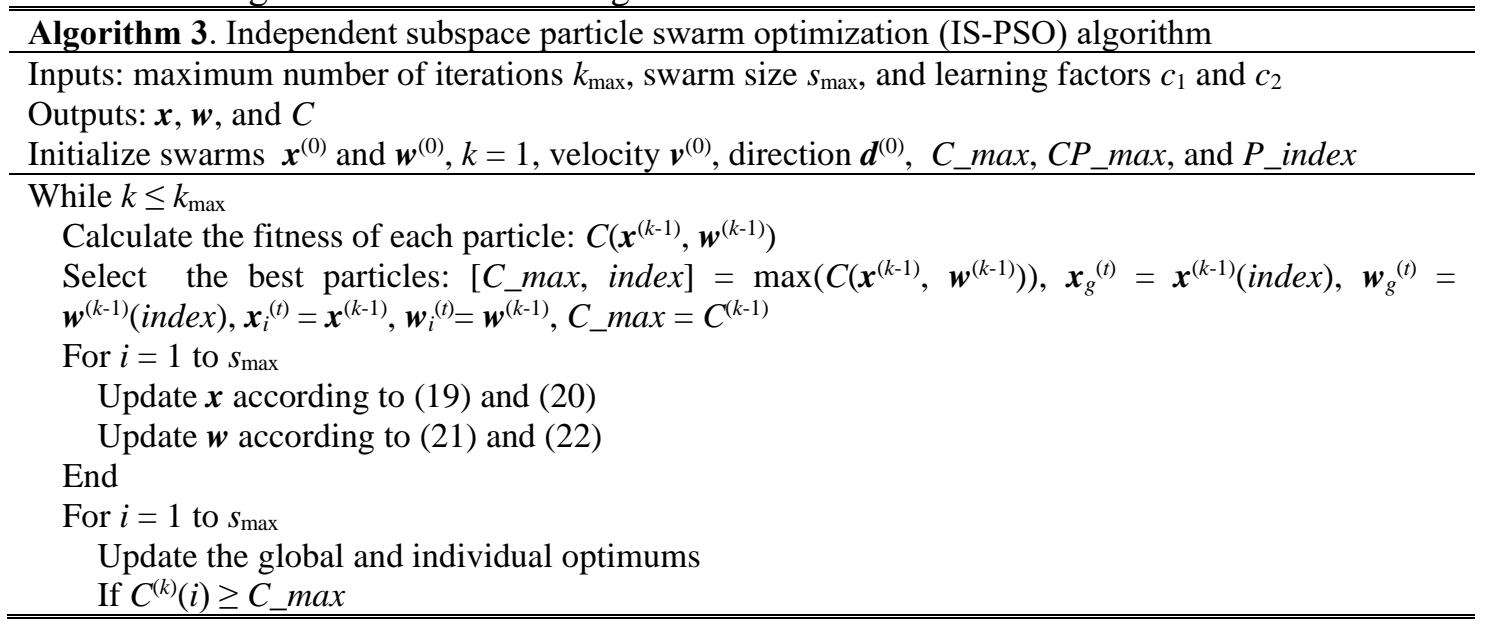




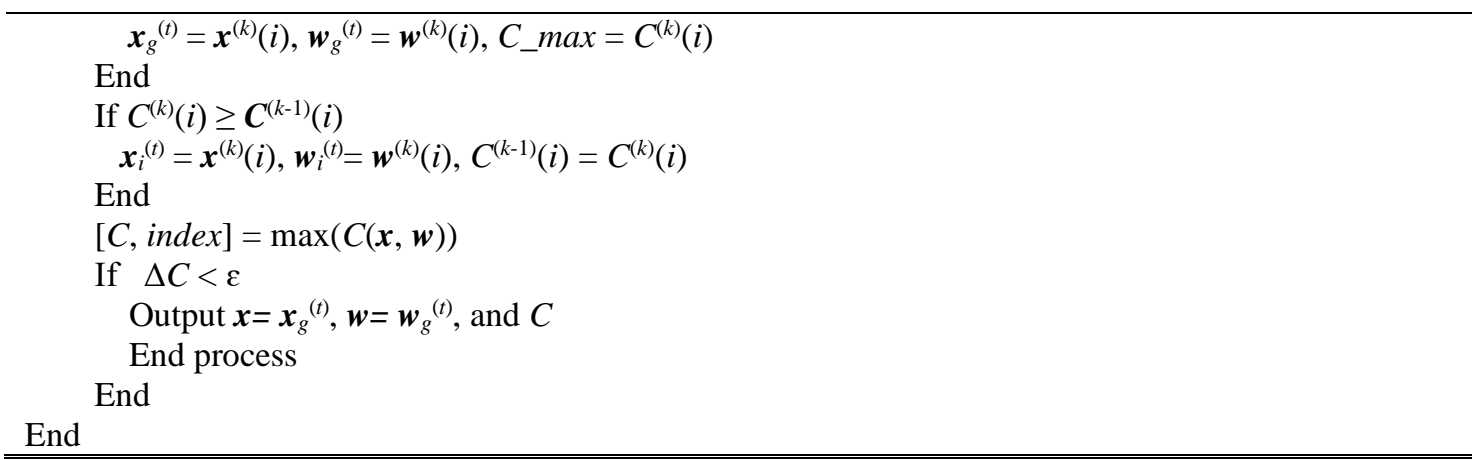

\subsubsection{Double Genetic Algorithm}

The double genetic algorithm (DGA) is decomposing (14) into subproblems involving $\boldsymbol{x}$ and $\boldsymbol{w}$, and select crossover or mutation for optimal solutions along the random position with respect to $\boldsymbol{x}$ and $\boldsymbol{w}$ until the total gradient $\Delta C$ is less than an acceptable error value $\varepsilon>0$. The DGA is defined in Algorithm 4.

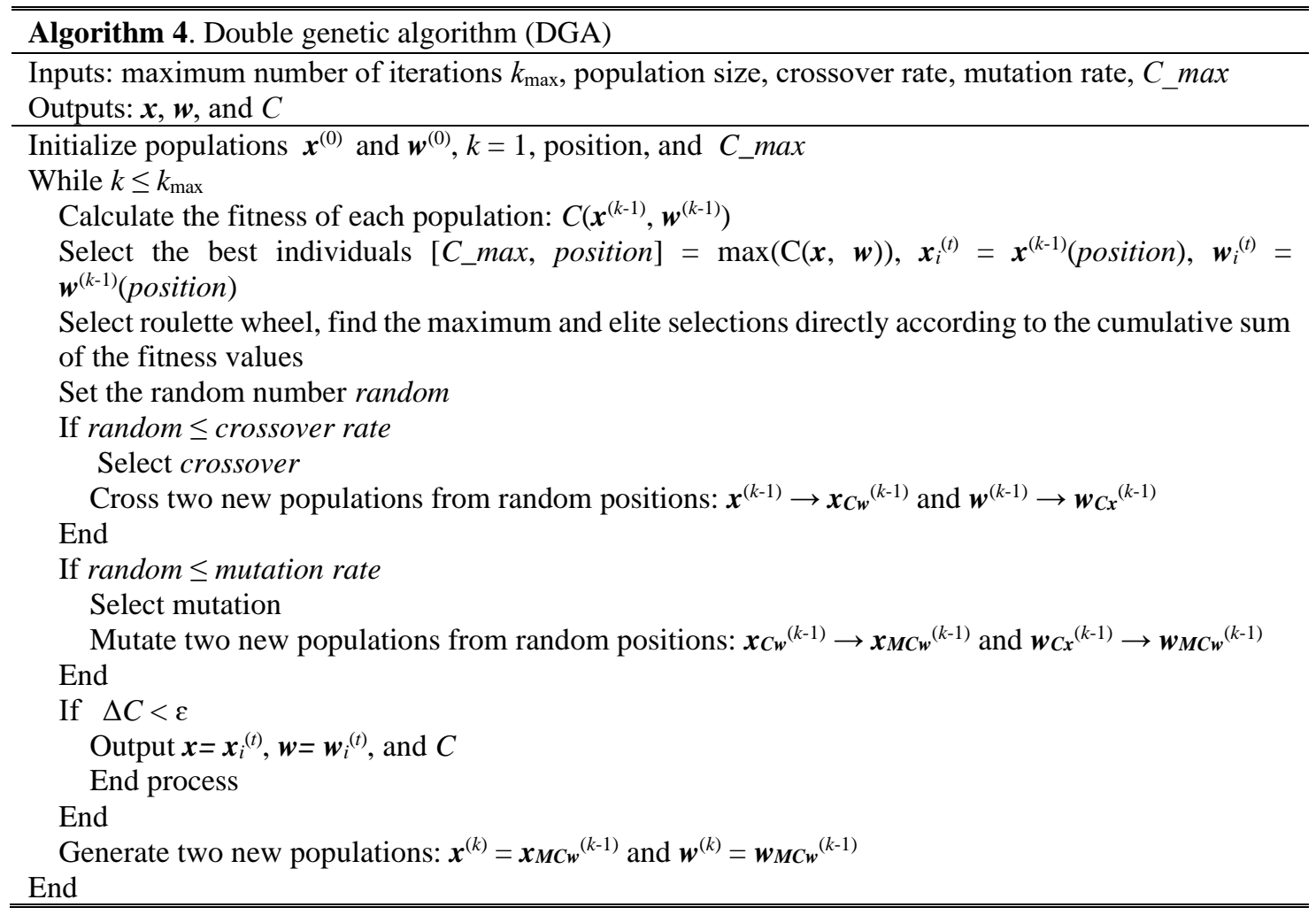

\subsubsection{Greedy Algorithm with Penalty Function}

The greedy algorithm with penalty function (GRA-PF) is decomposing (14) into subproblems involving $\boldsymbol{x}$ and $\boldsymbol{w}$, and select the penalty function for optimal solutions along the throughput with respect to $\boldsymbol{x}$ and $\boldsymbol{w}$ until the total gradient $\Delta C$ is less than an acceptable error value $\varepsilon>0$. Here, the penalty function is $B(\boldsymbol{w})=\sum \ln w_{i}\left(1-w_{i}\right)$, which is employed to penalize the throughput as $C^{\prime}=C+r B(\boldsymbol{w})$. The GRA-PF is defined in Algorithm 5 . 


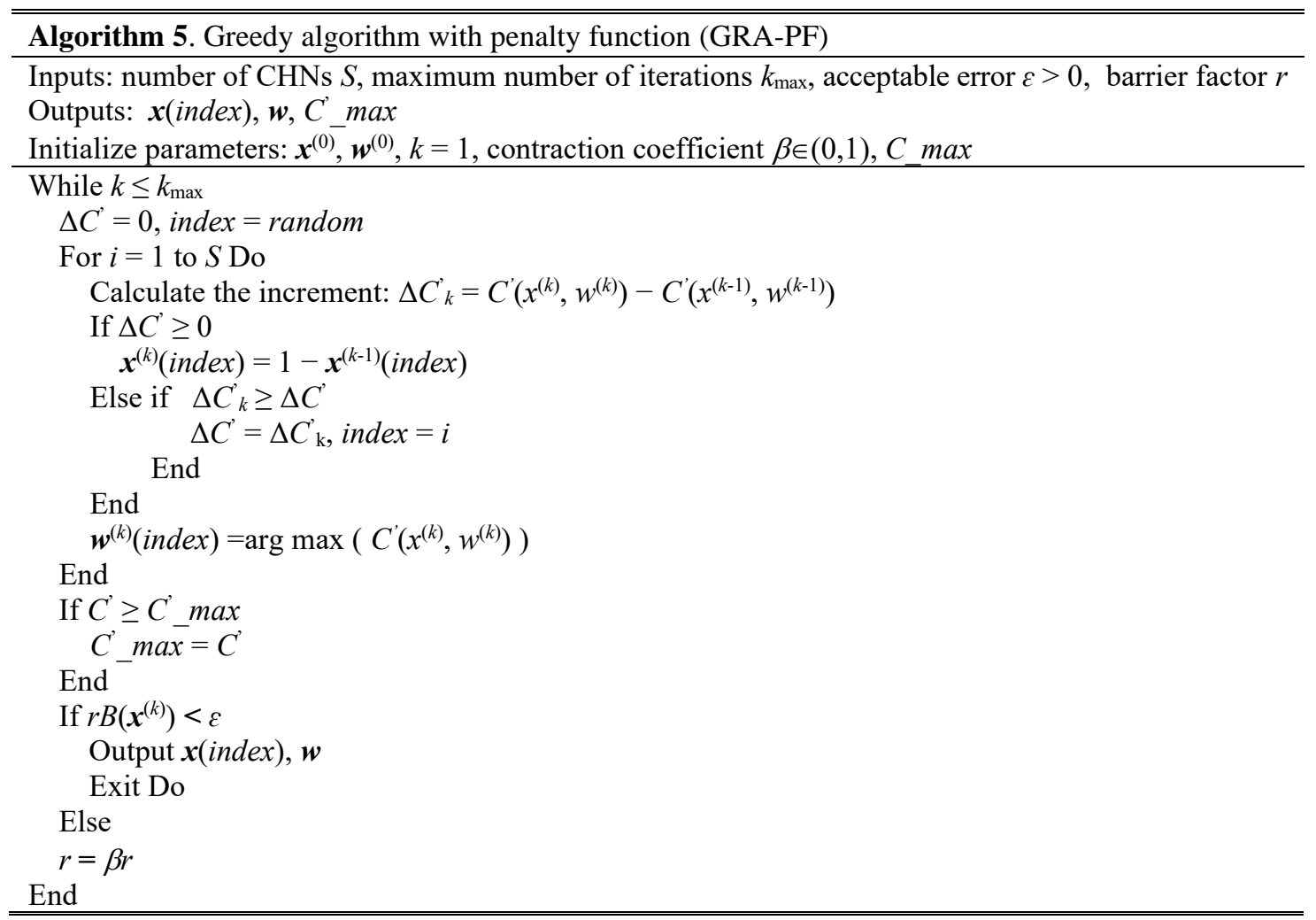

\section{Experimental Results and Analysis}

\subsection{Simulation Approach}

The effectiveness of applying the proposed power allocation with interference coordination method presented in Section 3 to the multi-robot network presented in Section 2 is validated through numerical investigations and event-driven simulations using MATLAB ${ }^{\circledR}$. Specifically, we first consider the effect of the number of MIMO antennas $N$ employed in the CPN of the network on network throughput for a network composed of 100 robot entities configured in 10 equally sized clusters, with $N$ in the range of $[2,32]$. We then consider the effect of the number of robot clusters employed in the network on network throughput for a network composed of 200 robot entities configured in generally equally sized clusters varying in number within the range of $[10,40]$ in increments of 5, with $N=32$. In addition, the results obtained with the different optimization methods presented in Section 4 for solving the problem are compared, and their respective advantages and disadvantages are analyzed. The simulations employ multiple robot entities and entity clusters moving randomly based on an RWP model according to the 3D spatial distribution and the technical characteristics of multiple robot networks. Meanwhile, the order of the internal actions of the network is based on existing head-cluster rules, which ensures network organization and the avoidance of collisions between robot entities. In terms of the communication channels, the path loss, Doppler frequency shifts, and power fade effects under LoS conditions are fully considered, and the jamming power $P_{d}$ obeys the normal distribution of the target transmission power. The specific parameters employed in the simulations are listed in Table 1. 
Table 1. Parameters employed in the simulations

\begin{tabular}{|c|c|}
\hline Parameter & Value \\
\hline Maximum coverage of CPNs, $R$ & 1000 feet \\
\hline Maximum coverage of CMNs, $r$ & 100 feet \\
\hline Carrier frequency, $f_{d}$ & $1.215 \mathrm{GHz}$ \\
\hline Bandwidth, $B$ & $20 \mathrm{MHz}$ \\
\hline Transmission power of CPNs, $P_{\mathrm{CP}}$ & $68 \mathrm{dBm}$ \\
\hline Transmission power of CMNs, $P_{\mathrm{CM}}$ & $53 \mathrm{dBm}$ \\
\hline Noise spectral density & $-129 \mathrm{dBm} / \mathrm{Hz}$ \\
\hline Maximum Robot speed, $v_{c}$ & 15 feet $/ \mathrm{s}$ \\
\hline Avoidance radius, $r$ & 10 feet \\
\hline
\end{tabular}

\subsection{Verification Analyses}

\subsubsection{Effect of Number of MIMO Antennas on Network Throughput}

The effect of the value of $N$ on network throughput, the proportion of CHNs operating in the IBFD mode, and the total weights of power allocation are presented in Fig. 4, Fig. 5, and Fig. 6, respectively, when the number of robots is 100 with 10 equally sized robot clusters.

The results in Fig. 4 indicate that the network throughput increases with increasing $N$, but that the increase is periodic, with poorly configured numbers of antennas presenting approximately zero throughput. This is because the poorly configured numbers of antennas suffer from self-interference. Eliminating the self-interference between antennas for large-scale MIMO antenna configurations is an important research topic. In addition, the optimum network throughput obtained by the various optimization algorithms considered are generally similar, except that the IS-SD algorithm performs most poorly within the peak periods obtained in the range of $N$ from 22 to 32 .

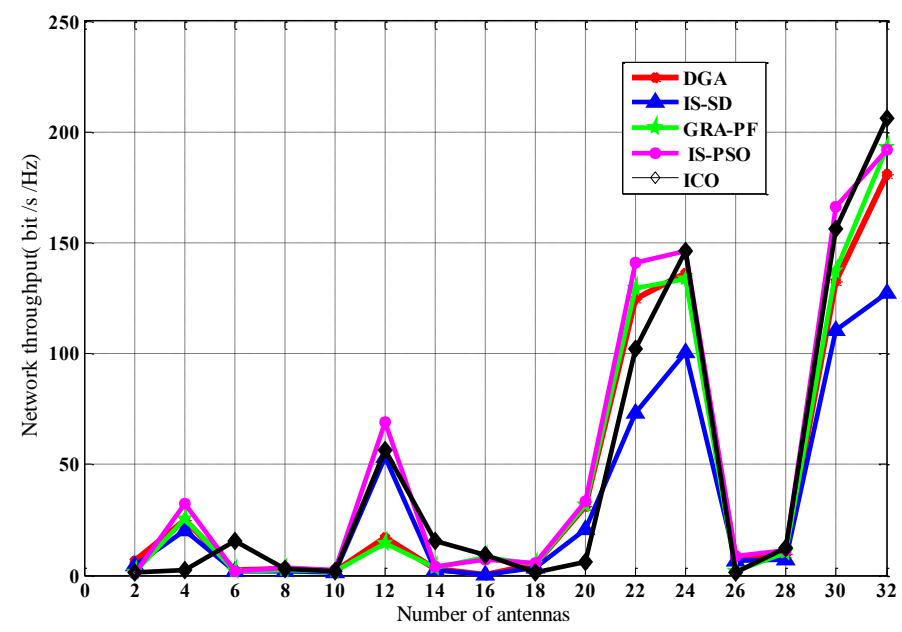

Fig. 4. Network throughput obtained by the various optimization algorithms with respect to the number of MIMO antennas employed for the CPN of the multi-robot network when the number of robots is 100 with 10 equally sized robot clusters 


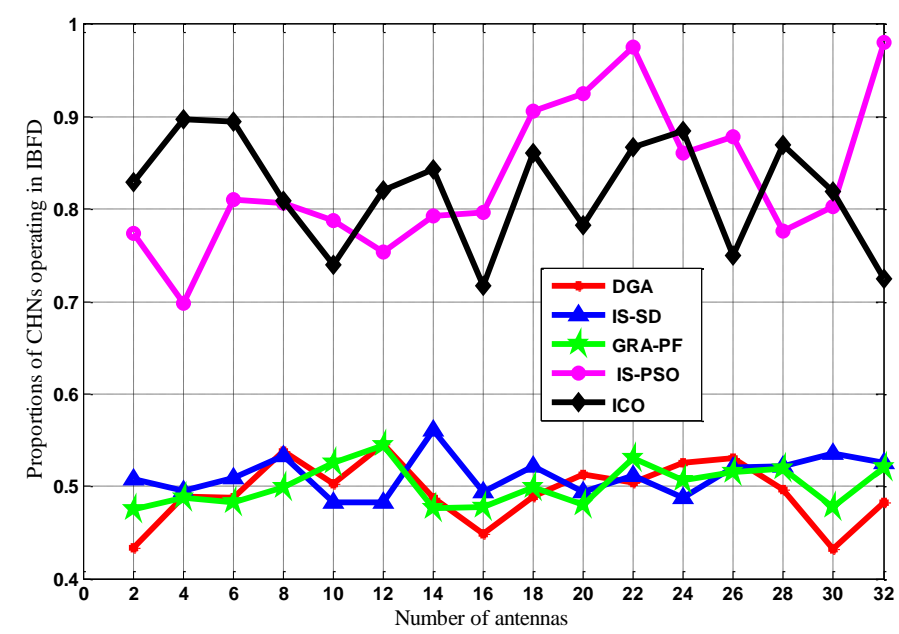

Fig. 5. Proportions of CHNs in the IBFD mode assigned by the various optimization algorithms with respect to the number of MIMO antennas employed for the CPN when the number of robots is 100 with 10 equally sized robot clusters

However, the IS-PSO and ICO algorithms are found to be superior to the other optimization algorithms in terms of the proportion of CHNs operating in the IBFD mode (Fig. 5) and the weights of power allocation (Fig. 6). Here, we note that the IS-PSO and ICO algorithms are found to assign considerably higher proportions of CHNs to the IBFD mode, which achieves about $60 \%$ improvement than the other algorithms], while the assigned weights of power allocation are more energy efficient, particularly in the range of $N$ from 22 to 32. At the same time, the proportion of CHNs in the IBFD mode is found to vary little with respect to the value of $N$. Accordingly, the number of MIMO antennas has little effect on the selection of full-duplex modes. This is because the MIMO antennas are deployed on the CPN in the proposed network, and that has less effect on the overall interference coordination. Above all, the results illustrate that the value of $\mathrm{N}$ should be selected reasonably according to the network configuration. The results can be analyzed in detail by investigating which of the 10 CHNs were assigned to the IBFD mode and their individual weights of power allocation for $N=32$. These results further demonstrate the advantages of the ICO and IS-PSO algorithms, where these algorithms clearly assign more CHNs to the IBFD mode and apply smaller weights of power allocation for most of the CHNs compared with the other optimization algorithms. 


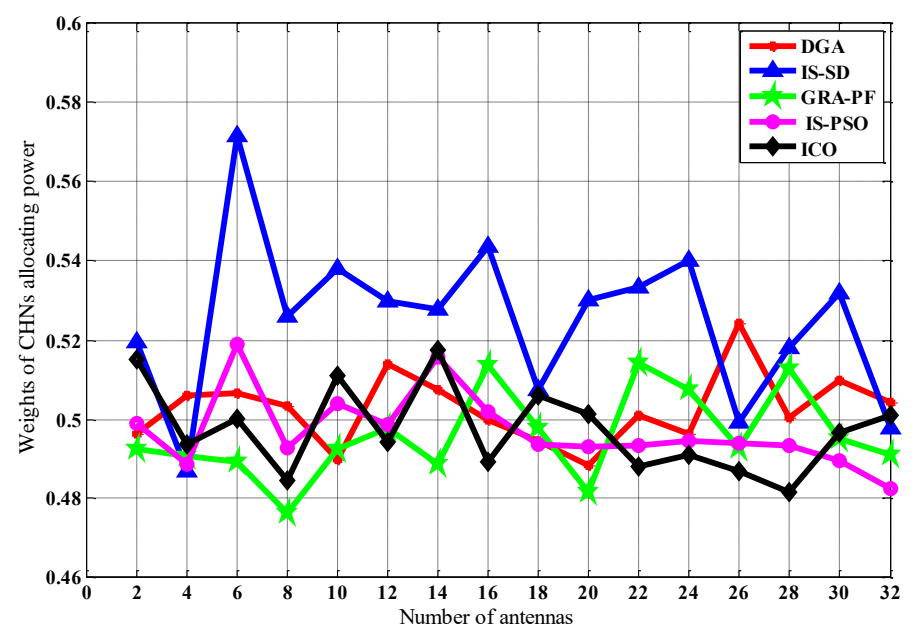

Fig. 6. Total weights of power allocation assigned to CHNs by the various optimization algorithms with respect to the number of MIMO antennas employed for the CPN when the number of robots is 100 with 10 equally sized robot clusters

\subsubsection{Effect of Number of Robot Clusters on Network Throughput}

The effect of the number of robot clusters on network throughput, the proportion of CHNs operating in the IBFD mode, and the total weights of power allocation are presented in Fig. 7, Fig. 8, and Fig. 9, respectively, when the number of robots is 200 with $N=32$. The results in Fig. 7 indicate that the emergent effect of the robot clusters becomes prominent when the number of CHNs lies within the range of $[30,35]$, which is about $13.04 \%-14.89 \%$ of the total number of robots in the network including the number of CHNs. Therefore, increasing the number of clusters to a certain extent can increase the overall network throughput. However, internal interference becomes a severe problem for further increases in the number of clusters, which increases the difficulty of coordinating network communication, and seriously restricts the overall communication capability of the network. Accordingly, applications of multi-robot networks require that a balance should be preserved between robot deployment density and communication capacity.

In addition, we again note that the IS-PSO and ICO algorithms are superior to the other optimization algorithms in terms of the proportion of CHNs operating in the IBFD mode, Fig. $\mathbf{8}$, and that the proportion of CHNs operating in this mode increases gradually with an increasing number of CHNs. Finally, we note from Fig. 9 that all of the considered optimization algorithms assigned fairly uniform total weights of power allocation regardless of the number of CHNs, except for the IS-SD algorithm, which again performed poorly for the most part. Furthermore, the FFD modes and weights of power allocation assigned by the optimization algorithms were analyzed in greater detail for a multi-robot network composed of 400 robots and $40 \mathrm{CHNs}$ with $N=32$ by investigating which of the $40 \mathrm{CHNs}$ were assigned to the IBFD mode and their individual weights of power allocation. These results further demonstrate the advantages of the ICO and IS-PSO algorithms compared with the other optimization algorithms. 


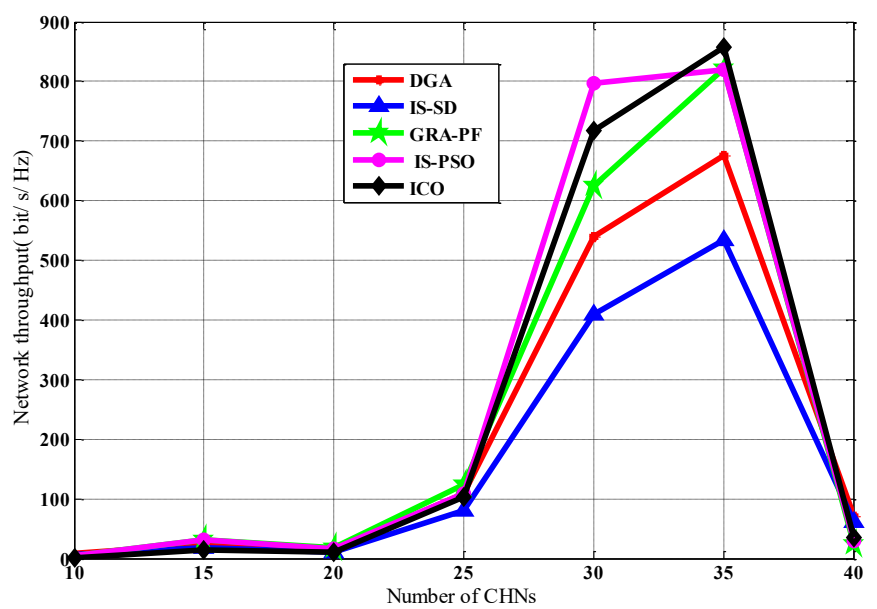

Fig. 7. Network throughput obtained by the various optimization algorithms with respect to the number of CHNs employed in the multi-robot network when the number of robots is 200 with $N=32$

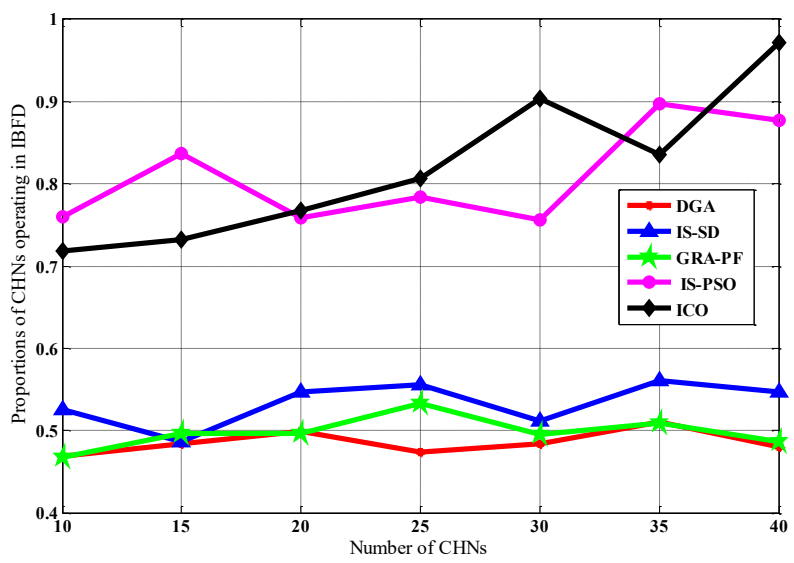

Fig. 8. Proportions of CHNs assigned to the IBFD mode by the various optimization algorithms with respect to the number of CHNs when the number of robots is 200 with $N=32$

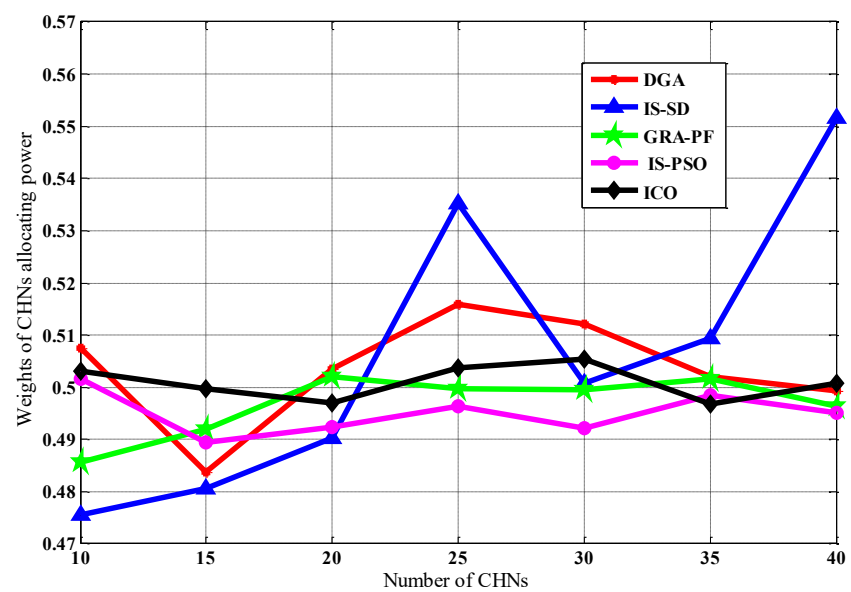

Fig. 9. Total weights of power allocation assigned by the various optimization algorithms with respect to the number of CHNs when the number of robots is 200 with $N=32$ 


\subsubsection{Computational Complexity of the Optimization Algorithms}

The computation times required by the various optimization algorithms for solving the proposed power allocation with interference coordination problem when the number of robots in the multi-robot network is 400, the number of CHNs $S$ varies from 10 to 70, and $N=32$ are shown in Fig. 10. We note that the GRA-PF algorithm requires an inordinately long time to solve the problem, while the SI-SD algorithm provides a very rapid solution at small values of $\mathrm{S}$ owing to the simplicity of the steepest descent method, but the computation time increases rapidly with increasing $S$. In contrast, the ICO and IS-PSO algorithms require relatively small computation times to solve the problem, particularly at high values of $S$, which vary from between $50 \%$ to $75 \%$ of the computation time required by the DGA algorithm. We note that the time complexity of the five optimization algorithms considered is uniformly $\mathrm{O}\left(S^{3}\right)$ when the number of robots remains constant. Yet, the ICO algorithm has a faster convergence rate, and therefore generally requires less computation time than the other algorithms considered.

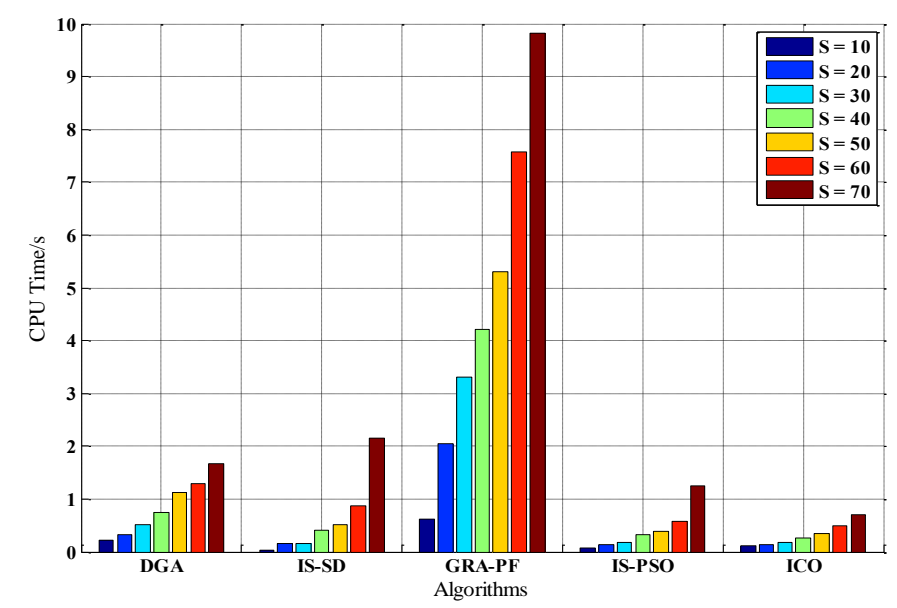

Fig. 10. Computation time required by the various optimization algorithms for solving the proposed power allocation with interference coordination problem when the number of robots in the multi-robot network is 400, the number of CHNs $S$ varies from 10 to 70 , and $N=32$

\section{Conclusion}

The present work addressed the challenging problem of coordinating power allocation with interference management in multi-robot networks by proposing a two-tier group-centric hierarchical framework that applies MIMO technology and full duplex systems for maximizing the throughput of networks under the impacts of Doppler frequency shifts and external jamming. The proposed power allocation with interference coordination formulation accounts for three types of the interference, including cross-tier, co-tier, and mixed-tier interference signals with CHNs operating in both OBFD and IBFD modes, and their SNRs were respectively derived under the impacts of Doppler frequency shifts and external jamming. In addition, five optimization algorithms were applied for solving the combined power allocation and interference coordination. The iterative callback optimization algorithm was determined to provide the best optimization performance based on its computational speed and the high performance of the obtained network. It is hoped that the presented models will help pave the way for future researchers to design multi-robot networks with ultra-high communication capacities. 


\section{References}

[1] E. F. Flushing, M. Kudelski, L. M. Gambardella, and G. A. Di Caro, "Connectivity-aware planning of search and rescue missions," in Proc. of 2013 IEEE International Symposium on Safety, Security, and Rescue Robotics, pp. 1-8, Oct. 2013. Article (CrossRef Link)

[2] S. Hayat, E. Yanmaz, T. X. Brown, and C. Bettstetter, "Multi-objective UAV path planning for search and rescue," in Proc. of 2017 IEEE International Conference on Robotics and Automation, pp. 5569-5574, June 2017. Article (CrossRef Link)

[3] L. Sabattini, C. Secchi, N. Chopra, and A. Gasparri, "Distributed control of multirobot systems with global connectivity maintenance," IEEE Transactions on Robotics, vol. 29, no. 5, pp. 1326-1332, Oct. 2013. Article (CrossRef Link)

[4] H. Ju and R. Zhang, "Throughput maximization in wireless powered communication networks," IEEE Transactions on Wireless Communications, vol. 13, no. 1, pp. 418-428, Jan. 2014. Article (CrossRef Link)

[5] N. Cheng, W. Xu, W. Shi, Y. Zhou, N. Lu, H. Zhou, and X. Shen, "Air-ground integrated mobile edge networks: Architecture, challenges, and opportunities," IEEE Communications Magazine, vol. 56, no. 8, pp. 26-32, Aug. 2018. Article (CrossRef Link)

[6] T. Long, M. Ozger, O. Cetinkaya, and O. B. Akan, "Energy neutral internet of drones," IEEE Communications Magazine, vol. 56, no. 1, pp. 22-28, Jan. 2018. Article (CrossRef Link)

[7] N. Kariminejad, S. A. Taher, M. Shahidehpour, and K. Khateri, "A hierarchical governor/turbine and electric vehicles optimal control framework for primary frequency support in power systems," IEEE Transactions on Smart Grid, vol. 9, no. 6, pp. 6702-6712, Nov. 2018.

Article (CrossRef Link)

[8] K. Khateri, M. Pourgholi, M. Montazeri, and L. Sabattini, "A comparison between decentralized local and global methods for connectivity maintenance of multi-robot networks," IEEE Robotics and Automation Letters, vol. 4, no. 2, pp. 633-940, Apr. 2019. Article (CrossRef Link)

[9] D. V. Dimarogonas and K. H. Johansson, "Bounded control of network connectivity in multi-agent systems," IET Control Theory \& Applications, vol. 4, no. 8, pp. 1330-1338, Aug. 2010. Article (CrossRef Link)

[10] O. S. Oubbati, M. Atiquzzaman, T. A. Ahanger, and A. Ibrahim, "Softwarization of UAV networks: A survey of applications and future trends," IEEE Access, vol. 8, pp. 98073-98125, May 2020. Article (CrossRef Link)

[11] J. Yao, W. Lou, C. Yang, and K. Wu, "Efficient interference-aware power control in wireless ad hoc networks," in Proc. of 2017 IEEE International Conference on Communications, pp. 1-6, May 2017. Article (CrossRef Link)

[12] A. Bezzina, M. Ayari, R. Langar, G. Pujolle, and L. Saidane, "Interference-aware game-based channel assignment algorithm for MR-MC WMNs,” 2014 IFIP Wireless Days, pp. 1-6, Nov. 2014. Article (CrossRef Link)

[13] U. Sterner, S. Linder, J. Nilsson, and K. Fors, "Inter-network interference in synchronized cooperative broadcast systems," in Proc. of 2018 IEEE Military Communications Conference, pp. 1-9, Oct. 2018. Article (CrossRef Link)

[14] Y. Mao, C. You, J. Zhang, K. Huang, and K. B. Letaief, "A survey on mobile edge computing: The communication perspective," IEEE Communications Surveys \& Tutorials, vol. 19, no. 4, pp. 2322-2358, 2017. Article (CrossRef Link)

[15] M. Li, N. Cheng, J. Gao, Y. Wang, L. Zhao, and X. Shen, "Energy-efficient UAV-assisted mobile edge computing: Resource allocation and trajectory optimization," IEEE Transactions on Vehicular Technology, vol. 69, no. 3, pp. 3424-3438, Mar. 2020. Article (CrossRef Link)

[16] W. Wang, X. Wang, and A. A. Nilsson, "Energy-efficient bandwidth allocation in wireless networks: Algorithms, analysis, and simulations," IEEE Transactions on Wireless Communications, vol. 5, no. 5, pp. 1103-1114, May 2006. Article (CrossRef Link)

[17] B. Alzahrani, O. Sami Oubbati, A. Barnawi, M. Atiquzzaman, and D. Alghazzawi, "UAV assistance paradigm: State-of-the-art in applications and challenges," Journal of Network and Computer Applications, vol. 166, pp.1-46, May 2020. Article (CrossRef Link) 
[18] R. Verdone and S. Mignardi, "Joint aerial-terrestrial resource management in UAV-aided mobile radio networks," IEEE Network, vol. 32, no. 5, pp. 70-75, Sep. 2018. Article (CrossRef Link)

[19] O. S. Oubbati, A. Lakas, P. Lorenz, M. Atiquzzaman, and A. Jamalipour, "Leveraging communicating UAVs for emergency vehicle guidance in urban areas," IEEE Transactions on Emerging Topics in Computing, July 2019. Article (CrossRef Link)

[20] Q. Wu, L. Liu, and R. Zhang, "Fundamental trade-offs in communication and trajectory design for UAV-enabled wireless network," IEEE Wireless Communications, vol. 26, no. 1, pp. 36-44, Feb. 2019. Article (CrossRef Link)

[21] G. Xu, Y. Li, J. Yuan, R. Monroe, S. Rajagopal, S. Ramakrishna, Y. Nam, J. Seol, J. Kim, M. M. U. Gul, A. Aziz, and J. Zhang, "Full dimension MIMO (FD-MIMO): Demonstrating commercial feasibility," IEEE Journal on Selected Areas in Communications, vol. 35, no. 8, pp. 1876-1886, Aug. 2017. Article (CrossRef Link)

[22] S. Biswas, K. Singh, O. Taghizadeh, and T. Ratnarajah, "Coexistence of MIMO radar and FD MIMO cellular systems with QoS considerations," IEEE Transactions on Wireless Communications, vol. 17, no. 11, pp. 7281-7294, Nov. 2018. Article (CrossRef Link)

[23] F. Heliot, M. A. Imran, and R. Tafazolli, "On the energy efficiency-spectral efficiency trade-off over the MIMO Rayleigh fading channel," IEEE Transactions on Communications, vol. 60, no. 5, pp. 1345-1356, May 2012. Article (CrossRef Link)

[24] H. Ahn, J. Lee, C. Kim, and Y. Suh, "Frequency domain coordination MAC protocol for full-duplex wireless networks," IEEE Communications Letters, vol. 23, no. 3, pp. 518-521, Mar. 2019. Article (CrossRef Link)

[25] S. Chen, T. Huang, K. C. Lin, Y. P. Hong, and A. Sabharwal, "Probabilistic medium access control for full-duplex networks with half-duplex clients," IEEE Transactions on Wireless Communications, vol. 16, no. 4, pp. 2627-2640, Apr. 2017. Article (CrossRef Link)

[26] T. P. Do and Y. H. Kim, "Resource allocation for a full-duplex wireless-powered communication network with imperfect self-interference cancelation," IEEE Communications Letters, vol. 20, no. 12, pp. 2482-2485, Dec. 2016. Article (CrossRef Link)

[27] S. Bandyopadhyay, E. J. Coyle, and T. Falck, "Stochastic properties of mobility models in mobile Ad hoc networks," IEEE Transactions on Mobile Computing, vol. 6, no. 11, pp. 1218-1229, Nov. 2007. Article (CrossRef Link)

[28] S. A. Hadiwardoyo, J. M. Dricot, C. T. Calafate, J. C. Cano, E. Hernández-Orallo, and P. Manzoni, "UAV mobility model for dynamic UAV-to-car communications in 3D environments," Ad Hoc Networks, vol. 107, Apr. 2020. Article (CrossRef Link)

[29] M. Schwartz, Mobile Wireless Communications, New York, NY, USA: Cambridge University Press, 2004. Article (CrossRef Link)

[30] Z. Zhang and W. Lyu, "Interference coordination in full-duplex HetNet with large-scale antenna arrays," Frontiers of Information Technology \& Electronic Engineering, vol. 18, no. 6, pp. 830-840, June 2017. Article (CrossRef Link)

[31] Y. Dong, F. Liao, T. Pang, H. Su, J. Zhu, X. Hu, and J. Li, "Boosting adversarial attacks with momentum," in Proc. of 2018 IEEE/CVF Conference on Computer Vision and Pattern Recognition, pp. 9185-9193, June 2018. Article (CrossRef Link)

[32] J. Smoczek and J. Szpytko, "Particle swarm optimization-based multivariable generalized predictive control for an overhead crane," IEEE/ASME Transactions on Mechatronics, vol. 22, no. 1, pp. 258-268, Feb. 2017. Article (CrossRef Link)

[33] Y. Li, W. Shao, L. You, and B. Wang, "An Improved PSO Algorithm and Its Application to UWB Antenna Design," IEEE Antennas and Wireless Propagation Letters, vol. 12, pp. 1236-1239, Sep. 2013. Article (CrossRef Link) 

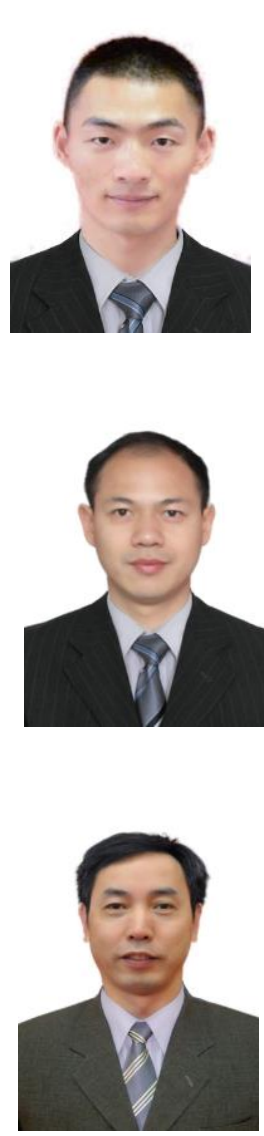

Guisheng Wang received the M.S. degree in information and communication engineering from the Information and Navigation College, Air Force Engineering University (AFEU), Xi'an, China, in 2018, where he is currently pursuing the Ph.D. degree in information and communication engineering. His research interests include military aeronautical communication and cognitive anti-jamming communication.

Yequn Wang received the M.S. and Ph.D. degrees from Air Force Engineering University (AFEU), in 2009 and 2012, respectively, where he is a currently a Lecturer with the Information and Navigation College. His main research interests include aeronautical communication, ad hoc networks, satellite communication, and HF communication.
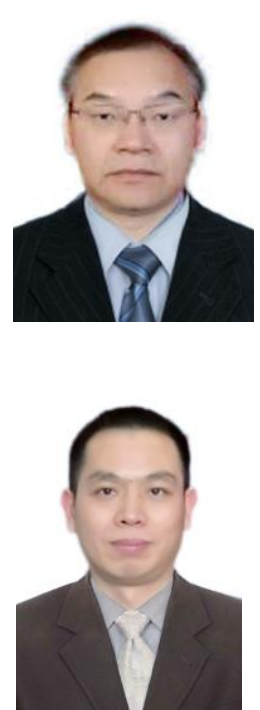

Shufu Dong received the M.S. degree from Air Force Engineering University (AFEU), in 1997, and the Ph.D. degree from the Xi'an Institute of Optics and Precision Mechanics, Chinese Academy of Science, Xi' an, China, in 2005. He is currently a Professor with the Information and Navigation College, Air Force Engineering University (AFEU). His main research interests include aeronautical communication and communication networking.

Guoce Huang received the M.S. degree from Air Force Telecommunication Engineering College, Xi'an, China, in 1990. He is currently a Doctoral Supervisor and a Professor with the Information and Navigation College, Air Force Engineering University (AFEU). His main research interests include $\mathrm{HF}$ communication, aeronautical communication, and satellite communication.

Qilu Sun received the M.S. and Ph.D. degrees from North Western Polytechnic University (NWPU), Xi'an, China, in 2005 and 2011, respectively. He is currently an associate Professor with the Information and Navigation College, Air Force Engineering University (AFEU). His main research interests include HF communication and aeronautical communication. 